\title{
Collaborative approach and lessons learnt from transitioning to remotely invigilated online examinations
}

\author{
Vinh Tran, Justin Chu and Jasmine Cheng \\ Insearch Limited Education
}

\begin{abstract}
The global pandemic in 2020 has spurred the rapid transition to remote learning for higher education providers in terms of course delivery and assessments. Final examinations which play an important role in summarily assessing students' attainments of course intended learning outcomes are traditionally conducted in a physical invigilated environment. This paper discussed the transition from paper-based physically invigilated final examinations into remotely invigilated online examinations. It highlights the collaborative approach across different parts of the organisation to bring together a smooth experience for students to undertake their final examinations while the academic integrity of the examinations is still maintained. It also sheds lights on some measures of the success of the transition including feedback from teaching staff and students who participated in the process from which lessons are learnt as feedforward for future undertakings.
\end{abstract}

Keywords: final examinations; remote learning; online examinations; remote invigilation; academic integrity

\section{Background}

UTS Insearch is the premium pathway provider to the University of Technology Sydney (UTS). The academic programs were conducted remotely since March 2020 as part of the institution's response to the COVID-19 pandemic. Our teaching staff and students were enthusiastic and appreciative that teaching and learning were not interrupted despite the pandemic. The focus on how to conduct classes remotely has shifted quickly to how to assess students effectively and authentically in an online environment. The remotely invigilated online examinations were conducted for the first time from 1 June 2020 to 5 June 2020. In remotely invigilated online examinations students bring their own device (BYOD), sit their examinations remotely and student recordings are invigilated post examinations (Cramp et al., 2019). According to Cramp et al. (2019), this type of examinations requires systematic and effective design compared to traditional paper-based examinations and should be supplemented by early and clear communication with students. Cramp et al. also suggest that more effort is required to set up the examinations and technical support will be essential for academic as well as students.

Universities have switched to online examinations during COVID-19 pandemic. We have poked around what other institutions are doing with examinations and found that University of Sydney, Western Sydney University, University of Technology Sydney are using similar proctoring solution for online examinations. One of the challenges in the remotely invigilated online examinations is the effectiveness of the examinations and how to maintain academic integrity and standards. Even though there are no foolproof ways to protect the integrity of examinations, there are certainly best practices and techniques that can be put in place to reduce the risk of academic misconduct.

We recognise that this is a stressful time for students as well as teaching staff. As teaching has gone online, many students have faced challenges including studying in a different environment, experiencing difficult conditions and are expected to have the hardware, software and Internet access to take the online examinations. To be operational in an online environment, the assessments that work well in face-to-face setting may not be effective under the online environment and may need to be adjusted or replaced. Frankl and Bitter (2012) found that academic staff needed support during the preparation of the online examinations, and that students also needed technical support during the examinations to minimise anxiety from sitting examinations in a new environment. This paper will outline the need and importance of implementing best practices and the procedures associated with the online examinations and shares the lesson learnt while moving to the remotely invigilated online examinations.

\section{The collaborative approach}

During the deliberations to arranging final examinations, key considerations were emphasised on enabling students to demonstrate mastery of learning outcomes to continue with their intended study plans as well as protecting the integrity of the final examinations. Special arrangements also needed to be considered with students on the Access 
and Inclusion register. This called for engagement and contribution from different parts of the organisation, from Information Technology and Digital Services (ITDS) staff, Examinations department, Study Success Advisors to teaching staff and students. There were several streams of work that needed to put in place to devise an entirely new examinations process designed for remote invigilation.

\section{Technology platforms}

Extensive research was performed into technological platforms that enable final examinations to be conducted online in a secure and reliable manner. UTS Insearch made the move to a new learning management system, Canvas, in 2019 which provided a user-friendly platform to create online examinations. Various technologies were considered in terms of usability for both teaching staff and students as well as economies of scale.

Lockdown Browser, which is an add-on tool integrated into Canvas, locks the browser into a single screen and prevents students from printing, copying, or accessing other applications during the examinations. Students are required to use Respondus Monitor that builds upon the Lockdown Browser. It uses a student's webcam and advanced video analytics to monitor examinations sessions. We have already been using Lockdown Browser for almost a year hence the further inclusion and requirement of Respondus Monitor was a natural next step for teaching staff and students when there is no new system to be learnt. The amalgamation of Lockdown Browser and Respondus Monitor provides a secure environment for students to undertake the final examinations and generates post-examinations reports that allow examinations staff to review students' activities throughout the examinations.

\section{A revised examinations invigilation process}

Given the high-stake nature of the final examinations and to provide timely support to students during the examinations, a new process was developed by Examinations department in consultation with Education staff and ITDS staff. Firstly, it was decided that an extra identity check was required for all students before the examinations could be taken. Examinations department reviewed each student's identity via Zoom with UTS Insearch student card or other forms of photo identification ensuring only verified students were accessing the examinations. This pre-examinations check also included students scanning environment around the room ensuring only authorised materials were accessible during the examinations. Secondly, throughout the duration of the examinations, examinations department maintained a live connection with students via Zoom to regularly check up on students as well as provided students with support during the examinations where necessary. Issues reported from students during the examinations were captured in a central location for documentation purposes as well as ensuring adequate considerations were given to students in special circumstances. Once the examinations were finished, the examinations teams reviewed reports from Respondus Monitor to identify and review high-risk cases to validate if misconducts occurred. A review of the reported issues also took place and special circumstances were accommodated via either assessment adjustments or resit for special examinations including through other means where Lockdown Browser proved to be not usable by students.

\section{Communication, training and support for staff}

All subject coordinators were communicated about the arrangement of the online examinations via email as well as one-to-one meetings with their program managers. To ensure all subject coordinators had the same level of understanding of the online examinations, each of them was provided with an online examinations protocol. The protocol explains the rationale behind, specifies the key dates, details the examinations procedures, and importantly, is a training guide itself for subject coordinators to create the online examinations in Canvas. In addition to the technical setup (such as, LockDown Browser and Respondus Monitor), the training guide highlights critical functionality in Canvas that allows the online examinations to be created, including randomising answer items for each question, selecting the overall question order and drawing questions from the question bank. All these allow subject coordinators to seamlessly create different examinations versions for the main, special and supplementary examinations, and importantly to ensure academic integrity. The guide also details how submission folders can be set up for students to upload their written work, authorised notes and rough-work sheets in Canvas. Overall, the training guide empowers subject coordinators to create different examinations settings to suit the subject needs in the online environment.

Each subject coordinator was given six weeks to set up online examinations. Two weeks before the examinations, a peer review exercise was conducted between subject coordinators. The review exercise involves cross-checking of the examinations settings and reviews on examinations question in terms of difficulty and suitability. The collaborative approach aims at sharing and learning the best practices between subject coordinators. The reviewed examinations were then sent to program managers for their final approval.

\section{Communication, training and support for students}

A series of actions were conducted to communicate, train and support students for the online examinations.

- Canvas and email messages: Students received five different messages about the online examinations 
throughout the semester via both email and Canvas announcement. The goal of these messages was to keep students informed and engaged with the online examinations, regarding the arrangement, preparation, key dates, procedure and support.

- Interactive FAQ guide: In Canvas, students were provided with access to an interactive FAQ guide on the online examinations. The guide was jointly prepared by the Education and Examinations departments. The overall goal of this FAQ guide is to address all possible concerns raised by students before the start of the online examinations. The FAQ explains what students should expect and be expected of before, during and after the online examinations. Throughout the guide, students are reminded with the support available and the key contact details if they have a concern and further questions. The guide also addresses the privacy concern by stating the type of data collected and explained how they were treated.

- Examinations support session: In each subject, students were invited to attend a one-hour examinations support session organised by the subject coordinator. The session aimed to provide students with the subject-specific information, such as the permitted materials for the examinations, the resources available for preparing the online examinations, and how students submit their work online. Students were also given opportunities to raise questions and concern.

- Examinations support workshop: Three weeks before the examinations, students were invited to attend a onehour examinations support workshop. The workshop was run by Study Success Advisors, Program Managers and the Examinations Coordinator. The workshop aimed to address general issues related to the examinations, such as study tips, technical troubleshooting, support and privacy concern.

- Practice examinations: Before the online examinations, compulsory practice examinations were organised for all students. The intent was to allow students to experience the online examinations process, including the identity authentication process, answering the questions online, submitting handwritten answer sheets, and interacting with the examinations invigilators.

- Technical support: The ITDS staff has been proactively reached out to students to provide technical support. Students who were not able to afford a laptop or had persistent internet connectivity issues were directed to the Welfare Team. The Team then provided these students with laptops and WIFI Dongles for the online examinations.

- Individual arrangements: Those students who are on the access and inclusion register or whose home environment was not suitable for the online examinations received alternative arrangements. These students took their online examinations in the computer labs at the campus under the supervision by the Study Success Advisors.

\section{Findings}

There were 2,490 examinations expected to be completed across 28 subjects and $95 \%$ of the examinations were successfully conducted without any issues. 133 issues were reported to ITDS and Examinations department, most of which were about technical issues encountered during the examinations. These students were provided accommodations such as special examinations or completion of examinations on a Microsoft Word document with live Zoom invigilation from Examinations department. 10 students completed their examinations on campus in cases where their home environment was not appropriate for online examinations. Social distancing rules were applied. After thorough invigilation and post-examinations check by the Examinations team, 4 misconduct cases were reported for the online examinations, compared to 7, 15 and 24 misconducts being reported in the prior three semesters conducted in a physically invigilated environment.

A survey about online examinations was also conducted with subject coordinators and students. With a $96 \%$ response rate from subject coordinators, $80 \%$ indicated that they were satisfied with the support and overall experience in the online examinations. They appreciated the collaborative approach to final examinations design and cross checking. They also highlighted some concern around more effective communication between the Examinations department and subject coordinators. Below are some qualitative comments from the subject coordinators.

The examinations team was very helpful to run the final examinations on the day. It was also very helpful to have a colleague to check my final examinations details.

There was not enough communication between examinations office and subject coordinators. Subject coordinators did not know how the examinations was actually arranged.

Communication from Examinations Department regarding scheduling special examinations, could have been better. Subject Coordinators need to be informed when these take place.

With a response rate of $15 \%$ from students, $70 \%$ indicated that they were satisfied with the support and overall experience in the online examinations. Technical issues encountered during the examinations were the major concern by students. Some qualitative comments are below.

There was a technical error on the UTS Insearch side for my examinations. Otherwise, it was good. 
I was too worried about different kinds of IT problem before the test as I am not in SYD. Worry about the speed of network and internet control in China. Finally, IT problems happened.

It wasn't a good preparation for examinations because we need teachers to help more and reply faster as we don't have much support. All teachers worry about is people cheating and it's not nice and fair for students that actually need the help to prepare like me because I struggle to make time to study.

\section{Conclusions}

\section{Successes and Lessons learnt}

The experience of the recent implementation suggests that communication is key to success. The feedback surveys we received from students and subject coordinators suggested that there should be more communication between the Examinations department, the subject coordinators and students. Students were provided with adequate information for the preparation and the requirements of the online examinations. However, some students were still not clear on the procedures when joining and leaving the online examinations, and what to do when they experienced technical issues. Subject coordinators were concerned that students might face technical issues navigating the online examinations. We learnt that many of the subject coordinators' concerns and the issues that students experienced during the online examinations could have been eliminated if they were familiar with the requirements and procedures provided in the FAQ circulated prior to the examinations.

It was a merit for the subject coordinators to collaboratively cross-check each other's examinations settings and format in pairs. They also prepared and facilitated practice test for students prior to the online examinations. Many subject coordinators proactively organised examinations workshops to communicate the expectations of the online examinations, and most importantly to advise students on physical and mental wellbeing during the examinations period.

\section{Future directions}

Workshops on tips and tricks to create good questions for the online examinations will be organised for subject coordinators. The overall aim of the workshop is to facilitate subject coordinators to rethink and redesign their examinations questions to ensure they are optimised for the online environment. The workshops will involve subject coordinators from different disciplines to share their best practice in the design of good online examinations questions. Various question types, such as the multiple-choice questions, short-answer response, true-false questions, structured questions and case studies, will be included. At the same time, participants will be invited to bring some examples of their examinations questions used in Semester 1, 2020 to discuss whether modifications can be made to improve the quality as well as students' test-taking experience in the online environment.

\section{Limitations and future studies}

In this study, there are limitations that could lead to future investigations for improving the online examinations. The first limitation is the relatively low student response rate. This is attributed to the timing of the feedback survey being available to students. When the survey was open to students, at least a third of the total student population who was in their final stage at UTS Insearch had already moved onto UTS to start their second year. One solution to this is to embed the online examinations survey in the actual online examinations so that more students' views can be captured.

Secondly, the nature of this study was a quantitative one, identifying adequacy of the support, preparation and communication for the online examinations. While some qualitative comments were collected in the survey, rich and in-depth qualitative data would shed light on the precise ways how support, preparation and communication could impact student learning experience in the online examinations. Therefore, in the future, we plan to collect views from different stakeholders, for example, subject coordinators from focus group interviews, and student representatives from the Student Advisory Groups.

\section{References}

Cramp, J., Medlin, J. F., Lake, P., \& Sharp, C. (2019). Lessons learned from implementing remotely invigilated online exams. Journal of University Teaching \& Learning Practice, 16(1), 10.

Frankl, G., \& Bitter, S. (2012, October). Online exams: practical implications and future directions. In Proceedings of the European Conference on e-Learning (pp. 158-164). 
Tran, V., Chu, J. \& Cheng, J. (2020). Collaborative approach and lessons learnt from transitioning to remotely invigilated online examinations. In S. Gregory, S. Warburton, \& M. Parkes (Eds.), ASCILITE's First Virtual

Conference. Proceedings ASCILITE 2020 in Armidale (pp. 301-305). https://doi.org/10.14742/ascilite2020.0127

Note: All published papers are refereed, having undergone a double-blind peer-review process.

The author(s) assign a Creative Commons by attribution licence enabling others to distribute, remix, tweak, and build upon their work, even commercially, as long as credit is given to the author(s) for the original creation.

(C) Tran, V., Chu, J. \& Cheng, J. 2020 